\title{
Regularity of Functions on the Reduced Quaternion Field in Clifford Analysis
}

\author{
Ji Eun Kim, Su Jin Lim, and Kwang Ho Shon \\ Department of Mathematics, Pusan National University, Busan 609-735, Republic of Korea \\ Correspondence should be addressed to Kwang Ho Shon; khshon@pusan.ac.kr
}

Received 11 December 2013; Revised 12 February 2014; Accepted 18 February 2014; Published 20 March 2014

Academic Editor: Junesang Choi

Copyright (C) 2014 Ji Eun Kim et al. This is an open access article distributed under the Creative Commons Attribution License, which permits unrestricted use, distribution, and reproduction in any medium, provided the original work is properly cited.

We define a new hypercomplex structure of $\mathbb{R}^{3}$ and a regular function with values in that structure. From the properties of regular functions, we research the exponential function on the reduced quaternion field and represent the corresponding Cauchy-Riemann equations in hypercomplex structures of $\mathbb{R}^{3}$.

\section{Introduction}

Meglihzon [1], Sudbery [2], and Fueter [3] demonstrated that there are three possible approaches (the Cauchy approach, Weierstrass approach, and Riemann approach) in the theories of functions that would generalize holomorphic functions with respect to several complex variables. Sudbery [2], Soucek [4], and Sommen [5] attempted to research the Cauchy approach using differential forms and differential operators in Clifford analysis. Fueter [3] and Naser [6] studied the properties of quaternionic differential equations as a generalization of the extended Cauchy-Riemann equations in the complex holomorphic function theory. Nôno [7-9] and Sudbery [2] gave a definition and the development of regular functions over the quaternion field. Ryan [10,11] developed the theories of regular functions in a complex Clifford analysis using a generalization of the Cauchy-Riemann equation. Malonek [12] considered analogously the function theory of hypercomplex variables. He defined the hypercomplex differentiability for the existence of a function over the Clifford algebra and monogenicity based on a generalized Cauchy-Riemann system. Gotô and Nôno [13] and Koriyama et al. [14] dealt with differential operators with the derivative of regular functions in quaternion.

We shall denote by $\mathbb{C}, \mathbb{R}$, and $\mathbb{Z}$, respectively, the field of complex numbers, the field of real numbers, and the set of all integers. We $[15,16]$ showed that any complexvalued harmonic function $f_{1}$ in a pseudoconvex domain
$D$ of $\mathbb{C}^{2} \times \mathbb{C}^{2}$ has a hyperconjugate harmonic function $f_{2}$ in $D$ such that the quaternion-valued function $f_{1}+f_{2} j$ is hyperholomorphic in $D$ and gave a regeneration theorem in quaternion analysis in the view of complex and Clifford analysis. Further, we $[17,18]$ investigated the existence of the hyperconjugate harmonic functions of the octonion number system and some properties of dual quaternion functions.

In this paper, we introduce the Fueter variables on $\mathbb{R}^{3}$ and investigate a hypercomplex structure of $\mathbb{R}^{3}$. We define regular functions and obtain the representation of the corresponding Cauchy-Riemann equations for regular functions in the reduced quaternion field.

\section{Preliminaries}

A three-dimensional, noncommutative, and associative real field, called a ternary number system, is constructed by three base elements $e_{0}, e_{1}$, and $e_{2}$ which satisfy

$$
\begin{array}{ll}
e_{0}^{2}=1, \quad e_{1}^{2}=e_{2}^{2}=-1, \quad e_{1} e_{2}=-e_{2} e_{1}, \\
\overline{e_{0}}=e_{0}, \quad \overline{e_{r}}=-e_{r} \quad(r=1,2) .
\end{array}
$$

In addition, let $e_{0}$ be the identity of a ternary number system and $e_{1}$ identifies the imaginary unit $\sqrt{-1}$ in the complex field, and

$$
\mathbb{C}(\mathbb{T}):=\left\{z=e_{1} z_{1}+e_{2} z_{2} \mid z_{1}, z_{2} \in \mathbb{C}\right\},
$$


where $z_{r}=x_{r}-(1 / 2) e_{r} x_{0}(r=1,2)$ and $x_{m}(m=0,1,2)$ are real variables. They satisfy the equations

$$
\overline{z_{r} w_{k}}=\overline{w_{k}} \overline{z_{r}} \quad(r \neq k),
$$

where $\overline{z_{r}}=x_{r}+(1 / 2) e_{r} x_{0}(r=1,2), w_{k}=y_{k}-(1 / 2) e_{k} y_{0}$, $\overline{w_{k}}=y_{k}+(1 / 2) e_{k} y_{0}(k=1,2)$, and $y_{m}(m=0,1,2)$ are real variables.

For any two elements $z=e_{1} z_{1}+e_{2} z_{2}$ and $w=e_{1} w_{1}+e_{2} w_{2}$ of $\mathbb{C}(\mathbb{\mathbb { T }})$, their product is given by

$$
z w=z \cdot w+z \odot w,
$$

where the corresponding commutative inner product $\bullet$ satisfies

$$
\begin{aligned}
z \cdot w & =\frac{1}{2}(z w+w z) \\
& =-\sum_{r=1}^{2} z_{r} w_{r}+\frac{1}{2} e_{1} e_{2}\left(\overline{z_{1}} w_{2}-\overline{w_{2}} z_{1}+\overline{w_{1}} z_{2}-\overline{z_{2}} w_{1}\right)
\end{aligned}
$$

and the corresponding noncommutative outer product $\odot$ satisfies

$$
\begin{aligned}
z \odot w & =\frac{1}{2}(z w-w z) \\
& =\frac{1}{2} e_{1} e_{2}\left(\overline{z_{1}} w_{2}+\overline{w_{2}} z_{1}-\overline{w_{1}} z_{2}-\overline{z_{2}} w_{1}\right) \\
& =-w \odot z .
\end{aligned}
$$

The conjugation $z^{*}$, the corresponding norm $|z|$, and the inverse $z^{-1}$ of $z$ in $\mathbb{C}(\mathbb{T})$ are given by

$$
\begin{gathered}
z^{*}=\overline{e_{1} z_{1}}+\overline{e_{2} z_{2}}, \\
|z|^{2}=z z^{*}=z \cdot z^{*}=\sum_{r=1}^{2} z_{r} \overline{z_{r}}, \\
z^{-1}=\frac{z^{*}}{|z|^{2}} \quad(z \neq 0) .
\end{gathered}
$$

For any element $z$ in $\mathbb{C}(\mathbb{T})$, we have the corresponding exponential function $e^{z}$ denoted by

$$
\exp (z)=\exp \left(e_{1} z_{1}+e_{2} z_{2}\right) .
$$

Theorem 1. Let $z$ be an arbitrary number in $\mathbb{C}(\mathbb{T})$. Then the corresponding exponential function $\exp (z)$ of $z$ in $\mathbb{C}(\mathbb{T})$ is given as

$$
\exp (z)= \begin{cases}(-1)^{k} \exp \left(x_{0}\right) \exp \left(e_{2} x_{2}\right), & \text { if } x_{1}=k \pi, \\ (-1)^{t} \exp \left(x_{0}\right) \exp \left(e_{1} x_{1}\right), & \text { if } x_{2}=t \pi,\end{cases}
$$

where $k, t \in \mathbb{Z}$.

Furthermore, as hyperbolic functions, one has

$\exp (z)$

$$
= \begin{cases}(-1)^{k} \exp \left(e_{2} x_{2}\right)\left(\cosh \left(x_{0}\right)-\sinh \left(x_{0}\right)\right), & \text { if } x_{1}=k \pi, \\ (-1)^{t} \exp \left(e_{1} x_{1}\right)\left(\cosh \left(x_{0}\right)-\sinh \left(x_{0}\right)\right), & \text { if } x_{2}=t \pi,\end{cases}
$$

where $k, t \in \mathbb{Z}$.
Proof. For any element $z=e_{1} z_{1}+e_{2} z_{2}$ of $\mathbb{C}(\mathbb{T})$,

$$
\exp (z)=\exp \left(e_{1} z_{1}+e_{2} z_{2}\right)=\exp \left(e_{1} z_{1}\right) \exp \left(e_{2} z_{2}\right) \text {. }
$$

Since a scalar part of $e_{1} z_{1}$ is $(1 / 2) x_{0}$, a vector part of $e_{1} z_{1}$ is $e_{1} x_{1}$, and $\left|e_{1}\right|=1$, by [19],

$$
\begin{aligned}
\exp \left(e_{1} z_{1}\right) & =\exp \left(\frac{x_{0}}{2}\right)\left\{\cos \left(\left|e_{1} x_{1}\right|\right)+\frac{e_{1} x_{1}}{\left|e_{1} x_{1}\right|} \sin \left(\left|e_{1} x_{1}\right|\right)\right\} \\
& =\exp \left(\frac{x_{0}}{2}\right)\left\{\cos \left(x_{1}\right)+e_{1} \sin \left(x_{1}\right)\right\}
\end{aligned}
$$

and, similarly, we have

$$
\begin{aligned}
\exp \left(e_{2} z_{2}\right) & =\exp \left(\frac{x_{0}}{2}\right)\left\{\cos \left(\left|e_{2} x_{2}\right|\right)+\frac{e_{2} x_{2}}{\left|e_{2} x_{2}\right|} \sin \left(\left|e_{2} x_{2}\right|\right)\right\} \\
& =\exp \left(\frac{x_{0}}{2}\right)\left\{\cos \left(x_{2}\right)+e_{2} \sin \left(x_{2}\right)\right\} .
\end{aligned}
$$

Then we have

$$
\begin{aligned}
\exp (z)= & \exp \left(\frac{x_{0}}{2}\right)\left\{\cos \left(x_{1}\right)+e_{1} \sin \left(x_{1}\right)\right\} \\
& \times \exp \left(\frac{x_{0}}{2}\right)\left\{\cos \left(x_{2}\right)+e_{2} \sin \left(x_{2}\right)\right\} \\
= & \exp \left(x_{0}\right)\left\{\cos \left(x_{1}\right)+e_{1} \sin \left(x_{1}\right)\right\} \\
& \times\left\{\cos \left(x_{2}\right)+e_{2} \sin \left(x_{2}\right)\right\} \\
= & \exp \left(x_{0}\right)\left\{\cos \left(x_{1}\right) \cos \left(x_{2}\right)+e_{2} \cos \left(x_{1}\right)\right. \\
& \left.\times \sin \left(x_{2}\right)+e_{1} \sin \left(x_{1}\right) \cos \left(x_{2}\right)\right\} \\
& +\exp \left(x_{0}\right) e_{1} e_{2} \sin \left(x_{1}\right) \sin \left(x_{2}\right) .
\end{aligned}
$$

Also, we obtain

$$
\begin{aligned}
\exp (z)= & \exp \left(e_{2} z_{2}+e_{1} z_{1}\right) \\
= & \exp \left(e_{2} z_{2}\right) \exp \left(e_{1} z_{1}\right) \\
= & \exp \left(x_{0}\right)\left\{\cos \left(x_{2}\right)+e_{2} \sin \left(x_{2}\right)\right\} \\
& \times\left\{\cos \left(x_{1}\right)+e_{1} \sin \left(x_{1}\right)\right\} \\
= & \exp \left(x_{0}\right)\left\{\cos \left(x_{1}\right) \cos \left(x_{2}\right)+e_{2} \cos \left(x_{1}\right)\right. \\
& \left.\quad \times \sin \left(x_{2}\right)+e_{1} \sin \left(x_{1}\right) \cos \left(x_{2}\right)\right\} \\
& +\exp \left(x_{0}\right) e_{2} e_{1} \sin \left(x_{1}\right) \sin \left(x_{2}\right) .
\end{aligned}
$$

Since (15) has to be equal to (14), $\sin \left(x_{1}\right) \sin \left(x_{2}\right)=0$, that is, $\sin \left(x_{1}\right)=0$ or $\sin \left(x_{2}\right)=0$. Therefore, $x_{1}=k \pi$ or $x_{2}=t \pi$, and then $\cos \left(x_{1}\right)=(-1)^{k}$ or $\cos \left(x_{2}\right)=(-1)^{t}$, where $k, t \in \mathbb{Z}$. If $x_{1}=k \pi(k \in \mathbb{Z})$, then

$$
\begin{aligned}
\exp (z) & =\exp \left(x_{0}\right)\left\{(-1)^{k}\left(\cos \left(x_{2}\right)+e_{2} \sin \left(x_{2}\right)\right)\right\} \\
& =(-1)^{k} \exp \left(x_{0}\right) \exp \left(e_{2} x_{2}\right) .
\end{aligned}
$$


Similarly, if $x_{2}=t \pi(t \in \mathbb{Z})$, then

$$
\begin{aligned}
\exp (z) & =\exp \left(x_{0}\right)\left\{(-1)^{t}\left(\cos \left(x_{1}\right)+e_{1} \sin \left(x_{1}\right)\right)\right\} \\
& =(-1)^{t} \exp \left(x_{0}\right) \exp \left(e_{1} x_{1}\right)
\end{aligned}
$$

Further, by the Euler formula and the addition rule of trigonometric functions,

$$
\begin{aligned}
\exp (z)= & \exp \left(e_{1} z_{1}+e_{2} z_{2}\right)=\exp \left(e_{1} z_{1}\right) \exp \left(e_{2} z_{2}\right) \\
= & \left(\cos \left(z_{1}\right)+e_{1} \sin \left(z_{1}\right)\right)\left(\cos \left(z_{2}\right)+e_{2} \sin \left(z_{2}\right)\right) \\
= & \left\{\cos \left(x_{1}\right) \cos \left(e_{1} \frac{x_{0}}{2}\right)+\sin \left(x_{1}\right) \sin \left(e_{1} \frac{x_{0}}{2}\right)\right. \\
& +e_{1}\left(\sin \left(x_{1}\right) \cos \left(e_{1} \frac{x_{0}}{2}\right)\right. \\
& \left.\left.-\cos \left(x_{1}\right) \sin \left(e_{1} \frac{x_{0}}{2}\right)\right)\right\} \\
& \quad\left\{\cos \left(x_{2}\right) \cos \left(e_{2} \frac{x_{0}}{2}\right)+\sin \left(x_{2}\right) \sin \left(e_{2} \frac{x_{0}}{2}\right)\right. \\
& +e_{2}\left(\sin \left(x_{2}\right) \cos \left(e_{2} \frac{x_{0}}{2}\right)\right. \\
& \left.\left.-\cos \left(x_{2}\right) \sin \left(e_{2} \frac{x_{0}}{2}\right)\right)\right\} .
\end{aligned}
$$

Since $\cos \left(e_{r}\left(x_{0} / 2\right)\right)=\cosh \left(x_{0} / 2\right)$ and $\sin \left(e_{r}\left(x_{0} / 2\right)\right)=$ $e_{r} \sinh \left(x_{0} / 2\right)(r=1,2)$, we have

$$
\begin{aligned}
& \exp (z)=\left\{\cos \left(x_{1}\right) \cosh \left(\frac{x_{0}}{2}\right)+e_{1} \sin \left(x_{1}\right) \sinh \left(\frac{x_{0}}{2}\right)\right. \\
&+e_{1}\left(\sin \left(x_{1}\right) \cosh \left(\frac{x_{0}}{2}\right)\right. \\
&\left.\left.-e_{1} \cos \left(x_{1}\right) \sinh \left(\frac{x_{0}}{2}\right)\right)\right\} \\
& \cdot\left\{\cos \left(x_{2}\right) \cosh \left(\frac{x_{0}}{2}\right)+e_{2} \sin \left(x_{2}\right) \sinh \left(\frac{x_{0}}{2}\right)\right. \\
& \quad+e_{2}\left(\sin \left(x_{2}\right) \cosh \left(\frac{x_{0}}{2}\right)\right. \\
&\left.\left.\quad-e_{2} \cos \left(x_{2}\right) \sinh \left(\frac{x_{0}}{2}\right)\right)\right\} \\
&=\left\{\left(\cos \left(x_{1}\right)+e_{1} \sin \left(x_{1}\right)\right)\right. \\
&\left.\quad \times\left(\cosh \left(\frac{x_{0}}{2}\right)-\sinh \left(\frac{x_{0}}{2}\right)\right)\right\} \\
& \times\left\{\left(\cos \left(x_{2}\right)+e_{2} \sin \left(x_{2}\right)\right)\right. \\
&\left.\quad \times\left(\cosh \left(\frac{x_{0}}{2}\right)-\sinh \left(\frac{x_{0}}{2}\right)\right)\right\}
\end{aligned}
$$

$$
\begin{aligned}
= & \left(\cos \left(x_{1}\right)+e_{1} \sin \left(x_{1}\right)\right)\left(\cos \left(x_{2}\right)+e_{2} \sin \left(x_{2}\right)\right) \\
& \times\left(\cosh \left(\frac{x_{0}}{2}\right)-\sinh \left(\frac{x_{0}}{2}\right)\right)^{2} .
\end{aligned}
$$

Since

$$
\begin{aligned}
& \left(\cosh \left(\frac{x_{0}}{2}\right)-\sinh \left(\frac{x_{0}}{2}\right)\right)^{2} \\
& =\cosh ^{2}\left(\frac{x_{0}}{2}\right)+\sinh ^{2}\left(\frac{x_{0}}{2}\right)-2 \cosh ^{2}\left(\frac{x_{0}}{2}\right) \sinh ^{2}\left(\frac{x_{0}}{2}\right) \\
& =1+2 \frac{\cosh \left(x_{0}\right)-1}{2}-\sinh \left(x_{0}\right) \\
& =\cosh \left(x_{0}\right)-\sinh \left(x_{0}\right),
\end{aligned}
$$

we obtain

$$
\begin{aligned}
\exp (z)= & \exp \left(e_{1} z_{1}\right) \exp \left(e_{2} z_{2}\right)=\left(\cos \left(x_{1}\right)+e_{1} \sin \left(x_{1}\right)\right) \\
& \times\left(\cos \left(x_{2}\right)+e_{2} \sin \left(x_{2}\right)\right)\left(\cosh \left(x_{0}\right)-\sinh \left(x_{0}\right)\right)
\end{aligned}
$$

and, similarly,

$$
\begin{aligned}
\exp (z)= & \exp \left(e_{2} z_{2}\right) \exp \left(e_{1} z_{1}\right)=\left(\cos \left(x_{2}\right)+e_{2} \sin \left(x_{2}\right)\right) \\
& \times\left(\cos \left(x_{1}\right)+e_{1} \sin \left(x_{1}\right)\right)\left(\cosh \left(x_{0}\right)-\sinh \left(x_{0}\right)\right) .
\end{aligned}
$$

Since (22) has to be equal to (21), $\sin \left(x_{1}\right) \sin \left(x_{2}\right)=0$, that is, $\sin \left(x_{1}\right)=0$ or $\sin \left(x_{2}\right)=0$. Therefore, $x_{1}=k \pi$ or $x_{2}=t \pi$, and then $\cos \left(x_{1}\right)=(-1)^{k}$ or $\cos \left(x_{2}\right)=(-1)^{t}$, where $k, t \in \mathbb{Z}$. If $x_{1}=k \pi(k \in \mathbb{Z})$, then

$$
\begin{aligned}
\exp (z)= & \left(\cos \left(x_{2}\right)+e_{2} \sin \left(x_{2}\right)\right) \\
& \times(-1)^{k}\left(\cosh \left(x_{0}\right)-\sinh \left(x_{0}\right)\right) \\
= & (-1)^{k} \exp \left(e_{2} x_{2}\right)\left(\cosh \left(x_{0}\right)-\sinh \left(x_{0}\right)\right) .
\end{aligned}
$$

Similarly, if $x_{2}=t \pi(t \in \mathbb{Z})$, then

$$
\begin{aligned}
\exp (z)= & \left(\cos \left(x_{1}\right)+e_{1} \sin \left(x_{1}\right)\right) \\
& \times(-1)^{t}\left(\cosh \left(x_{0}\right)-\sinh \left(x_{0}\right)\right) \\
= & (-1)^{t} \exp \left(e_{1} x_{1}\right)\left(\cosh \left(x_{0}\right)-\sinh \left(x_{0}\right)\right) .
\end{aligned}
$$

Remark 2. By Theorem 1 and the properties of the Euler formula, if $x_{1}=k \pi$, then we can write

$$
\begin{aligned}
\exp (z) & =(-1)^{k} \exp \left(e_{2} x_{2}\right)\left(\cosh \left(x_{0}\right)-\sinh \left(x_{0}\right)\right) \\
& =(-1)^{k} \exp \left(e_{2} x_{2}-x_{0}\right)=(-1)^{k} \exp \left(e_{2} \overline{F_{2}}\right),
\end{aligned}
$$


also, if $x_{2}=t \pi$, then

$$
\begin{aligned}
\exp (z) & =(-1)^{t} \exp \left(e_{1} x_{1}\right)\left(\cosh \left(x_{0}\right)-\sinh \left(x_{0}\right)\right) \\
& =(-1)^{t} \exp \left(e_{1} x_{1}-x_{0}\right)=(-1)^{t} \exp \left(e_{1} \overline{F_{1}}\right),
\end{aligned}
$$

where $k, t \in \mathbb{Z}$ and $\overline{F_{r}}=x_{r}+e_{r} x_{0}(r=1,2)$ are the conjugate Fueter variables of $F_{r}=x_{r}-e_{r} x_{0}(r=1,2)$ (see [20]).

Let $\Omega$ be an open subset of $\mathbb{R}^{3}$ and let a function $f(a)$ be defined by the following form on $\Omega$ with values in $\mathbb{C}(\mathbb{T})$ :

$$
f: \Omega \longrightarrow \mathbb{C}(\mathbb{T})
$$

satisfying

$$
\begin{aligned}
a= & \left(x_{0}, x_{1}, x_{2}\right) \in \Omega \longmapsto f(a)=e_{1} f_{1}\left(x_{0}, x_{1}, x_{2}\right) \\
& +e_{2} f_{2}\left(x_{0}, x_{1}, x_{2}\right) \in \mathbb{C}(\mathbb{T}),
\end{aligned}
$$

where $f_{r}=u_{r}-(1 / 2) e_{r} u_{0}, \overline{f_{r}}=u_{r}+(1 / 2) e_{r} u_{0}(r=1,2)$ and $u_{m}(m=0,1,2)$ are real-valued functions.

From the chain rule, we use the following differential operators:

$$
\begin{gathered}
\frac{\partial}{\partial A}:=2 \frac{\partial}{\partial x_{0}}-\frac{1}{2} e_{1} \frac{\partial}{\partial x_{1}}-\frac{1}{2} e_{2} \frac{\partial}{\partial x_{2}}=-e_{1} \frac{\partial}{\partial z_{1}}-e_{2} \frac{\partial}{\partial z_{2}} \\
\frac{\partial}{\partial A^{*}}=2 \frac{\partial}{\partial x_{0}}+\frac{1}{2} e_{1} \frac{\partial}{\partial x_{1}}+\frac{1}{2} e_{2} \frac{\partial}{\partial x_{2}}=e_{1} \frac{\partial}{\partial \overline{z_{1}}}+e_{2} \frac{\partial}{\partial \overline{z_{2}}}
\end{gathered}
$$

where

$$
\begin{array}{rlrl}
\frac{\partial}{\partial z_{1}}= & \frac{1}{2} \frac{\partial}{\partial x_{1}}+e_{1} \frac{\partial}{\partial x_{0}}, & \frac{\partial}{\partial z_{2}} & =\frac{1}{2} \frac{\partial}{\partial x_{2}}+e_{2} \frac{\partial}{\partial x_{0}}, \\
\frac{\partial}{\partial \overline{z_{1}}}=\frac{1}{2} \frac{\partial}{\partial x_{1}}-e_{1} \frac{\partial}{\partial x_{0}}, & \frac{\partial}{\partial \overline{z_{2}}}=\frac{1}{2} \frac{\partial}{\partial x_{2}}-e_{2} \frac{\partial}{\partial x_{0}}
\end{array}
$$

in $\mathbb{C}(\mathbb{T})$. We have the following equations:

$$
\begin{gathered}
f_{r} \frac{\partial}{\partial z_{r}}=\frac{\partial f_{r}}{\partial z_{r}}, \quad f_{r} \frac{\partial}{\partial \overline{z_{r}}}=\frac{\partial f_{r}}{\partial \overline{z_{r}}} \quad(r=1,2), \\
\overline{f_{1}} \frac{\partial}{\partial z_{2}}=\overline{\left(\frac{\partial f_{1}}{\partial \overline{z_{2}}}\right)}, \quad \overline{f_{2}} \frac{\partial}{\partial z_{1}}=\overline{\left(\frac{\partial f_{2}}{\partial \overline{z_{1}}}\right)} \\
\overline{f_{1}} \frac{\partial}{\partial \overline{z_{2}}}=\overline{\left(\frac{\partial f_{1}}{\partial z_{2}}\right)}, \quad \overline{f_{2}} \frac{\partial}{\partial \overline{z_{1}}}=\overline{\left(\frac{\partial f_{2}}{\partial z_{1}}\right)},
\end{gathered}
$$

and then, the operator $\partial / \partial A$ operates to $f$ as follows:

$$
\begin{aligned}
\frac{\partial f}{\partial A} & =\left(-e_{1} \frac{\partial}{\partial z_{1}}-e_{2} \frac{\partial}{\partial z_{2}}\right)\left(e_{1} f_{1}+e_{2} f_{2}\right) \\
& =\frac{\partial f_{1}}{\partial z_{1}}+\frac{\partial f_{2}}{\partial z_{2}}+e_{1} e_{2}\left(\frac{\partial f_{1}}{\partial \overline{z_{2}}}-\frac{\partial f_{2}}{\partial \overline{z_{1}}}\right),
\end{aligned}
$$

$$
\begin{aligned}
\frac{\partial f}{\partial A^{*}} & =\left(e_{1} \frac{\partial}{\partial \overline{z_{1}}}+e_{2} \frac{\partial}{\partial \overline{z_{2}}}\right)\left(e_{1} f_{1}+e_{2} f_{2}\right) \\
& =-\frac{\partial f_{1}}{\partial \overline{z_{1}}}-\frac{\partial f_{2}}{\partial \overline{z_{2}}}+e_{1} e_{2}\left(\frac{\partial f_{2}}{\partial z_{1}}-\frac{\partial f_{1}}{\partial z_{2}}\right) \\
f \frac{\partial}{\partial A} & =\left(e_{1} f_{1}+e_{2} f_{2}\right)\left(-e_{1} \frac{\partial}{\partial z_{1}}-e_{2} \frac{\partial}{\partial z_{2}}\right) \\
& =f_{1} \frac{\partial}{\partial z_{1}}+f_{2} \frac{\partial}{\partial z_{2}}+e_{1} e_{2}\left(\overline{f_{2}} \frac{\partial}{\partial z_{1}}-\overline{f_{1}} \frac{\partial}{\partial z_{2}}\right) \\
& =\frac{\partial f_{1}}{\partial z_{1}}+\frac{\partial f_{2}}{\partial z_{2}}+e_{1} e_{2}\left\{\overline{\left(\frac{\partial f_{2}}{\partial \overline{z_{1}}}\right)}-\overline{\left.\left(\frac{\partial f_{1}}{\partial \overline{z_{2}}}\right)\right\}}\right. \\
f \frac{\partial}{\partial A^{*}} & =\left(e_{1} f_{1}+e_{2} f_{2}\right)\left(e_{1} \frac{\partial}{\partial \overline{z_{1}}}+e_{2} \frac{\partial}{\partial \overline{z_{2}}}\right) \\
& =-f_{1} \frac{\partial}{\partial \overline{z_{1}}}-f_{2} \frac{\partial}{\partial \overline{z_{2}}}+e_{1} e_{2}\left(\overline{f_{1}} \frac{\partial}{\partial \overline{z_{2}}}-\overline{f_{2}} \frac{\partial}{\partial \overline{z_{1}}}\right) \\
& =-\frac{\partial f_{1}}{\partial \overline{z_{1}}}-\frac{\partial f_{2}}{\partial \overline{z_{2}}}+e_{1} e_{2}\left\{\overline{\left(\frac{\partial f_{1}}{\partial z_{2}}\right)}-\left(\frac{\partial f_{2}}{\partial z_{1}}\right)\right\}
\end{aligned}
$$

Thus, we have a corresponding Laplacian in the reduced quaternion $\mathbb{C}(\mathbb{T})$ :

$$
\Delta_{a}=\frac{\partial^{2}}{\partial A \partial A^{*}}=\frac{\partial^{2}}{\partial A^{*} \partial A}=4 \frac{\partial^{2}}{\partial x_{0}^{2}}+\frac{1}{4} \frac{\partial^{2}}{\partial x_{1}^{2}}+\frac{1}{4} \frac{\partial^{2}}{\partial x_{2}^{2}}
$$

Remark 3. Let $\Omega$ be an open set of $\mathbb{R}^{3}$. From the definition of the differential operators in $\mathbb{C}(\mathbb{T})$, we have

$$
\begin{aligned}
\frac{\partial}{\partial A} \bullet f= & \frac{1}{2}\left(\frac{\partial f_{1}}{\partial z_{1}}+\frac{\partial f_{2}}{\partial z_{2}}+f_{1} \frac{\partial}{\partial z_{1}}+f_{2} \frac{\partial}{\partial z_{2}}\right) \\
& +\frac{1}{2} e_{1} e_{2}\left(\overline{f_{2}} \frac{\partial}{\partial z_{1}}-\frac{\partial f_{2}}{\partial \overline{z_{1}}}+\frac{\partial f_{1}}{\partial \overline{z_{2}}}-\overline{f_{1}} \frac{\partial}{\partial z_{2}}\right) \\
= & \frac{\partial f_{1}}{\partial z_{1}}+\frac{\partial f_{2}}{\partial z_{2}}+\frac{1}{2} e_{1} e_{2} \\
& \times\left\{\overline{\left(\frac{\partial f_{2}}{\partial \overline{z_{1}}}\right)}-\overline{\left(\frac{\partial f_{1}}{\partial \overline{z_{2}}}\right)}-\frac{\partial f_{2}}{\partial \overline{z_{1}}}+\frac{\partial f_{1}}{\partial \overline{z_{2}}}\right\}, \\
\frac{\partial}{\partial A} \odot= & \frac{1}{2}\left(\frac{\partial f_{1}}{\partial z_{1}}+\frac{\partial f_{2}}{\partial z_{2}}-f_{1} \frac{\partial}{\partial z_{1}}-f_{2} \frac{\partial}{\partial z_{2}}\right) \\
& +\frac{1}{2} e_{1} e_{2}\left(\overline{f_{1}} \frac{\partial}{\partial z_{2}}+\frac{\partial f_{1}}{\partial \overline{z_{2}}}-\frac{\partial f_{2}}{\partial \overline{z_{1}}}-\overline{f_{2}} \frac{\partial}{\partial z_{1}}\right) \\
= & \frac{1}{2} e_{1} e_{2}\left\{\overline{\left(\frac{\partial f_{1}}{\partial \overline{z_{2}}}\right)}+\frac{\partial f_{1}}{\partial \overline{z_{2}}}-\frac{\partial f_{2}}{\partial \overline{z_{1}}}-\overline{\left(\frac{\partial f_{2}}{\partial \overline{z_{1}}}\right)}\right\},
\end{aligned}
$$




$$
\begin{aligned}
\frac{\partial}{\partial A^{*}} \cdot f= & -\frac{1}{2}\left(\frac{\partial f_{1}}{\partial \overline{z_{1}}}+\frac{\partial f_{2}}{\partial \overline{z_{2}}}+f_{1} \frac{\partial}{\partial \overline{z_{1}}}+f_{2} \frac{\partial}{\partial \overline{z_{2}}}\right) \\
& +\frac{1}{2} e_{1} e_{2}\left(\frac{\partial f_{2}}{\partial z_{1}}-\overline{f_{2}} \frac{\partial}{\partial \overline{z_{1}}}+\overline{f_{1}} \frac{\partial}{\partial \overline{z_{2}}}-\frac{\partial f_{1}}{\partial z_{2}}\right) \\
= & -\frac{\partial f_{1}}{\partial \overline{z_{1}}}-\frac{\partial f_{2}}{\partial \overline{z_{2}}}+\frac{1}{2} e_{1} e_{2} \\
& \times\left\{\frac{\partial f_{2}}{\partial z_{1}}-\overline{\left(\frac{\partial f_{2}}{\partial z_{1}}\right)}+\overline{\left(\frac{\partial f_{1}}{\partial z_{2}}\right)}-\frac{\partial f_{1}}{\partial z_{2}}\right\}, \\
\frac{\partial}{\partial A^{*}} \odot f= & \frac{1}{2}\left(-\frac{\partial f_{1}}{\partial \overline{z_{1}}}-\frac{\partial f_{2}}{\partial \overline{z_{2}}}+f_{1} \frac{\partial}{\partial \overline{z_{1}}}+f_{2} \frac{\partial}{\partial \overline{z_{2}}}\right) \\
& +\frac{1}{2} e_{1} e_{2}\left(\frac{\partial f_{2}}{\partial z_{1}}+\overline{f_{2}} \frac{\partial}{\partial \overline{z_{1}}}-\overline{f_{1}} \frac{\partial}{\partial \bar{z}_{2}}-\frac{\partial f_{1}}{\partial z_{2}}\right) \\
= & \frac{1}{2} e_{1} e_{2}\left\{\frac{\partial f_{2}}{\partial z_{1}}+\overline{\left(\frac{\partial f_{2}}{\partial z_{1}}\right)}-\overline{\left(\frac{\partial f_{1}}{\partial z_{2}}\right)}-\frac{\partial f_{1}}{\partial z_{2}}\right\}
\end{aligned}
$$

and, therefore,

$$
\frac{\partial f}{\partial A}=\frac{\partial}{\partial A} \cdot f+\frac{\partial}{\partial A} \odot f, \quad \frac{\partial f}{\partial A^{*}}=\frac{\partial}{\partial A^{*}} \cdot f+\frac{\partial}{\partial A^{*}} \odot f .
$$

Similarly, we have

$$
\begin{aligned}
& f \cdot \frac{\partial}{\partial A}=\frac{1}{2}\left(f_{1} \frac{\partial}{\partial z_{1}}+f_{2} \frac{\partial}{\partial z_{2}}+\frac{\partial f_{1}}{\partial z_{1}}+\frac{\partial f_{2}}{\partial z_{2}}\right) \\
& +e_{1} e_{2}\left(\overline{f_{1}} \frac{\partial}{\partial z_{2}}-\overline{f_{2}} \frac{\partial}{\partial z_{1}}+\frac{\partial f_{2}}{\partial \overline{z_{1}}}-\frac{\partial f_{1}}{\partial \overline{z_{2}}}\right) \\
& =\frac{\partial f_{1}}{\partial z_{1}}+\frac{\partial f_{2}}{\partial z_{2}}+\frac{1}{2} e_{1} e_{2} \\
& \times\left\{\overline{\left(\frac{\partial f_{1}}{\partial \overline{z_{2}}}\right)}-\overline{\left(\frac{\partial f_{2}}{\partial \overline{z_{1}}}\right)}+\frac{\partial f_{2}}{\partial \overline{z_{1}}}-\frac{\partial f_{1}}{\partial \overline{z_{2}}}\right\}, \\
& f \odot \frac{\partial}{\partial A}=\frac{1}{2}\left(f_{1} \frac{\partial}{\partial z_{1}}+f_{2} \frac{\partial}{\partial z_{2}}-\frac{\partial f_{1}}{\partial z_{1}}-\frac{\partial f_{2}}{\partial z_{2}}\right) \\
& +\frac{1}{2} e_{1} e_{2}\left(\overline{f_{1}} \frac{\partial}{\partial z_{2}}-\overline{f_{2}} \frac{\partial}{\partial z_{1}}-\frac{\partial f_{2}}{\partial \overline{z_{1}}}+\frac{\partial f_{1}}{\partial \overline{z_{2}}}\right) \\
& =\frac{1}{2} e_{1} e_{2}\left\{\overline{\left(\frac{\partial f_{1}}{\partial \overline{z_{2}}}\right)}-\overline{\left(\frac{\partial f_{2}}{\partial \overline{z_{1}}}\right)}-\frac{\partial f_{2}}{\partial \overline{z_{1}}}+\frac{\partial f_{1}}{\partial \overline{z_{2}}}\right\}, \\
& f \cdot \frac{\partial}{\partial A^{*}}=\frac{1}{2}\left(-f_{1} \frac{\partial}{\partial \overline{z_{1}}}-f_{2} \frac{\partial}{\partial \overline{z_{2}}}-\frac{\partial f_{1}}{\partial \overline{z_{1}}}-\frac{\partial f_{2}}{\partial \overline{z_{2}}}\right) \\
& +\frac{1}{2} e_{1} e_{2}\left(\overline{f_{1}} \frac{\partial}{\partial \overline{z_{2}}}-\overline{f_{2}} \frac{\partial}{\partial \overline{z_{1}}}+\frac{\partial f_{2}}{\partial z_{1}}-\frac{\partial f_{1}}{\partial z_{2}}\right) \\
& =-\frac{\partial f_{1}}{\partial \overline{z_{1}}}-\frac{\partial f_{2}}{\partial \overline{z_{2}}}+\frac{1}{2} e_{1} e_{2} \\
& \times\left\{\overline{\left(\frac{\partial f_{1}}{\partial z_{2}}\right)}-\overline{\left(\frac{\partial f_{2}}{\partial z_{1}}\right)}+\frac{\partial f_{2}}{\partial z_{1}}-\frac{\partial f_{1}}{\partial z_{2}}\right\},
\end{aligned}
$$

$$
\begin{aligned}
f \odot \frac{\partial}{\partial A^{*}}= & \frac{1}{2}\left(-f_{1} \frac{\partial}{\partial \overline{z_{1}}}-f_{2} \frac{\partial}{\partial \overline{z_{2}}}+\frac{\partial f_{1}}{\partial \overline{z_{1}}}+\frac{\partial f_{2}}{\partial \overline{z_{2}}}\right) \\
& +\frac{1}{2} e_{1} e_{2}\left(\overline{f_{1}} \frac{\partial}{\partial \overline{z_{2}}}-\overline{f_{2}} \frac{\partial}{\partial \overline{z_{1}}}-\frac{\partial f_{2}}{\partial z_{1}}+\frac{\partial f_{1}}{\partial z_{2}}\right) \\
= & \frac{1}{2} e_{1} e_{2}\left\{\overline{\left(\frac{\partial f_{1}}{\partial z_{2}}\right)}-\overline{\left(\frac{\partial f_{2}}{\partial z_{1}}\right)}-\frac{\partial f_{2}}{\partial z_{1}}+\frac{\partial f_{1}}{\partial z_{2}}\right\}
\end{aligned}
$$

and, therefore,

$$
\begin{gathered}
f \frac{\partial}{\partial A}=f \cdot \frac{\partial}{\partial A}+f \odot \frac{\partial}{\partial A}, \\
f \frac{\partial}{\partial A^{*}}=f \cdot \frac{\partial}{\partial A^{*}}+f \odot \frac{\partial}{\partial A^{*}} .
\end{gathered}
$$

Definition 4. Let $\Omega$ be an open set in $\mathbb{R}^{3}$ and for any element $a$ in $\Omega$. A function $f(a)$ is said to be $L(R)$-regular on $\Omega$ if the following conditions are satisfied:

(i) $f_{r}(r=1,2)$ are continuously differential functions on $\Omega$, and

(ii) $\partial f(a) / \partial A^{*}=0\left(f(a)\left(\partial / \partial A^{*}\right)=0\right)$ on $\Omega$.

In particular, the equation $\partial f / \partial A^{*}=0$ of Definition 4 is equivalent to

$$
\frac{\partial}{\partial A^{*}} \cdot f(a)=-\frac{\partial}{\partial A^{*}} \odot f(a) .
$$

Moreover, (38) is equivalent to the following system:

$$
\begin{gathered}
\frac{\partial f_{1}}{\partial \overline{z_{1}}}=-\frac{\partial f_{2}}{\partial \overline{z_{2}}}, \\
\frac{\partial f_{1}}{\partial z_{2}}=\frac{\partial f_{2}}{\partial z_{1}} .
\end{gathered}
$$

The above system is a corresponding Cauchy-Riemann system in $\mathbb{C}(\mathbb{T})$.

Remark 5. From the multiplications of $\mathbb{C}(\mathbb{T})$, the equation $f\left(\partial / \partial A^{*}\right)=0$ of Definition 4 is equivalent to

$$
\frac{\partial}{\partial A^{*}} \cdot f(a)=\frac{\partial}{\partial A^{*}} \odot f(a) .
$$

Also, the above equation (40) is equivalent to the following system:

$$
\begin{gathered}
\frac{\partial f_{1}}{\partial \overline{z_{1}}}=-\frac{\partial f_{2}}{\partial \overline{z_{2}}}, \\
\overline{f_{1}} \frac{\partial}{\partial \overline{z_{2}}}=\overline{f_{2}} \frac{\partial}{\partial \overline{z_{1}}} \Longleftrightarrow \overline{\left(\frac{\partial f_{1}}{\partial z_{2}}\right)}=\overline{\left(\frac{\partial f_{2}}{\partial z_{1}}\right) .}
\end{gathered}
$$

Further, the above system (41) is also a corresponding Cauchy-Riemann system in $\mathbb{C}(\mathbb{T})$. Since the system (39) is equivalent to the system (41), we say that $f(a)$ of Definition 4 is a regular function on $\Omega \subset \mathbb{R}^{3}$. When the function $f(a)$ is either an $L$-regular function or an $R$-regular function on $\Omega \subset \mathbb{R}^{3}$, we simply say that $f(a)$ is a regular function on $\Omega \subset \mathbb{R}^{3}$. 


\section{Properties of Regular Functions with Values in $\mathbb{C}(\mathbb{T})$}

We define the derivative $f^{\prime}(a)$ of $f(a)$ by the following:

$$
f^{\prime}(a):=\frac{\partial f(a)}{\partial A} .
$$

Proposition 6. Let $\Omega$ be an open set in $\mathbb{R}^{3}$ and let a function $f(a)$ be a regular function defined on $\Omega$. Then

$$
\begin{aligned}
f^{\prime}(a) & =-2 e_{r}\left(\frac{\partial f}{\partial z_{r}}-\frac{\partial f}{\partial \overline{z_{r}}}\right)=4 \frac{\partial f}{\partial x_{0}} \\
& =-e_{1} \frac{\partial f}{\partial x_{1}}-e_{2} \frac{\partial f}{\partial x_{2}} \quad(r=1,2) .
\end{aligned}
$$

Proof. From the definition of a regular function $\left(:\left(\partial f / \partial A^{*}\right)=0\right)$, we have

$$
\frac{\partial f_{1}}{\partial \overline{z_{1}}}=-\frac{\partial f_{2}}{\partial \overline{z_{2}}}, \quad \frac{\partial f_{1}}{\partial z_{2}}=\frac{\partial f_{2}}{\partial z_{1}} .
$$

Therefore,

$$
\begin{aligned}
& \frac{\partial}{\partial z} \cdot f=\frac{\partial f_{1}}{\partial \overline{z_{1}}}+2 e_{1} \frac{\partial u_{1}}{\partial x_{0}}+\frac{\partial u_{0}}{\partial x_{0}}+\frac{\partial f_{2}}{\partial \overline{z_{2}}}+2 e_{2} \frac{\partial u_{2}}{\partial x_{0}}+\frac{\partial u_{0}}{\partial x_{0}} \\
& +\frac{1}{2} e_{1} e_{2}\left(\overline{f_{2}} \frac{\partial}{\partial \overline{z_{1}}}+2 e_{1} \frac{\partial u_{2}}{\partial x_{0}}+e_{2} e_{1} \frac{\partial u_{0}}{\partial x_{0}}-\frac{\partial f_{2}}{\partial z_{1}}\right) \\
& +\frac{1}{2} e_{1} e_{2}\left(2 e_{1} \frac{\partial u_{2}}{\partial x_{0}}-e_{1} e_{2} \frac{\partial u_{0}}{\partial x_{0}}+\frac{\partial f_{1}}{\partial z_{2}}-2 e_{2} \frac{\partial u_{1}}{\partial x_{0}}\right. \\
& +e_{2} e_{1} \frac{\partial u_{0}}{\partial x_{0}}-\overline{f_{1}} \frac{\partial}{\partial \overline{z_{2}}} \\
& \left.-2 e_{2} \frac{\partial u_{1}}{\partial x_{0}}-e_{1} e_{2} \frac{\partial u_{0}}{\partial x_{0}}\right) \\
& =4 \frac{\partial f}{\partial x_{0}}+\frac{1}{2} e_{1} e_{2} \overline{\left(\frac{\partial f_{2}}{\partial z_{1}}\right)}-\frac{1}{2} e_{1} e_{2} \overline{\left(\frac{\partial f_{1}}{\partial z_{2}}\right)}, \\
& \frac{\partial}{\partial z} \odot f=\frac{1}{2} e_{1} e_{2} \\
& \times\left(\overline{f_{1}} \frac{\partial}{\partial \overline{z_{2}}}+2 e_{2} \frac{\partial f_{1}}{\partial x_{0}}+\frac{\partial f_{1}}{\partial z_{2}}-2 e_{2} \frac{\partial f_{1}}{\partial x_{0}}-\frac{\partial f_{2}}{\partial z_{1}}\right. \\
& \left.+2 e_{1} \frac{\partial f_{2}}{\partial x_{0}}-\overline{f_{2}} \frac{\partial}{\partial \overline{z_{1}}}-2 e_{1} \frac{\partial f_{2}}{\partial x_{0}}\right) \\
& =2 e_{1} e_{2}\left(e_{1} \frac{\partial f_{1}}{\partial x_{0}}-e_{1} \frac{\partial f_{1}}{\partial x_{0}}-e_{2} \frac{\partial f_{2}}{\partial x_{0}}+e_{2} \frac{\partial f_{2}}{\partial x_{0}}\right) \\
& -\frac{1}{2} e_{1} e_{2} \overline{\left(\frac{\partial f_{2}}{\partial z_{1}}\right)}+\frac{1}{2} e_{1} e_{2} \overline{\left(\frac{\partial f_{1}}{\partial z_{2}}\right)}=0 \text {. }
\end{aligned}
$$

Hence, we obtain the equation

$$
\frac{\partial f}{\partial z}=\frac{\partial}{\partial z} \cdot f+\frac{\partial}{\partial z} \odot f=4 \frac{\partial f}{\partial x_{0}} .
$$

Similarly, by calculating the derivative $f^{\prime}(z)$ of $f(z)$,

$$
\begin{aligned}
\frac{\partial}{\partial z} \cdot f= & \frac{\partial f_{1}}{\partial x_{1}}+\frac{\partial f_{2}}{\partial x_{2}} \\
& +\frac{1}{2} e_{1} e_{2}\left\{\overline{\left(\frac{\partial f_{2}}{\partial z_{1}}\right)}-\overline{\left(\frac{\partial f_{1}}{\partial z_{2}}\right)}+\frac{\partial f_{1}}{\partial x_{2}}-\frac{\partial f_{2}}{\partial x_{1}}\right\}, \\
\frac{\partial}{\partial z} \odot f= & \left(e_{1} \frac{\partial f_{2}}{\partial x_{1}}-e_{1} \frac{\partial f_{2}}{\partial x_{1}}-e_{2} \frac{\partial f_{1}}{\partial x_{2}}+e_{2} \frac{\partial f_{1}}{\partial x_{2}}\right) \\
& -\frac{1}{2} e_{1} e_{2} \overline{\left(\frac{\partial f_{2}}{\partial z_{1}}\right)}+\frac{1}{2} e_{1} e_{2} \overline{\left(\frac{\partial f_{1}}{\partial z_{2}}\right)} .
\end{aligned}
$$

Therefore, we have the equation

$$
\begin{aligned}
\frac{\partial f}{\partial z}= & \frac{\partial}{\partial z} \cdot f+\frac{\partial}{\partial z} \odot f=-e_{1}\left(e_{1} \frac{\partial f_{1}}{\partial x_{1}}+e_{2} \frac{\partial f_{2}}{\partial x_{1}}\right) \\
& -e_{2}\left(e_{2} \frac{\partial f_{2}}{\partial x_{2}}+e_{1} \frac{\partial f_{1}}{\partial x_{2}}\right)=-e_{1} \frac{\partial f}{\partial x_{1}}-e_{2} \frac{\partial f}{\partial x_{2}} .
\end{aligned}
$$

Further, using the same procedure, we obtain the equations

$$
\frac{\partial f}{\partial z}=-2 e_{r}\left(\frac{\partial f}{\partial z_{r}}-\frac{\partial f}{\partial \overline{z_{r}}}\right)(r=1,2) .
$$

Proposition 7. Let $\Omega$ be an open set in $\mathbb{R}^{3}$. If $f(a)$ is a regular function on $\Omega$, then we have

$$
\frac{\partial^{n} f}{\partial A^{n}}=4^{n} \frac{\partial^{n} f}{\partial x_{0}^{n}}
$$

where $n$ is a positive integer.

Proof. Since $f$ is a regular function on $\Omega$ with values in $\mathbb{C}(\mathbb{T})$, by Definition 4 ,

$$
\frac{\partial}{\partial A^{*}}\left(4 \frac{\partial f}{\partial x_{0}}\right)=4 \frac{\partial}{\partial x_{0}}\left(\frac{\partial f}{\partial A^{*}}\right)=0 .
$$

Hence, $\partial f / \partial x_{0}$ is a regular function with values in $\mathbb{C}(\mathbb{T})$. From Proposition 6, we have

$$
\frac{\partial^{2} f}{\partial A^{2}}=\frac{\partial}{\partial A}\left(\frac{\partial f}{\partial \mathrm{A}}\right)=4^{2} \frac{\partial^{2} f}{\partial x_{0}^{2}} .
$$

By repeating the above process, we can obtain the equation

$$
\frac{\partial^{n} f}{\partial A^{n}}=4^{n} \frac{\partial^{n} f}{\partial x_{0}^{n}}
$$

We let

$$
\square_{a}=\sum_{r=1}^{2} \frac{\partial^{2}}{\partial z_{r} \partial \overline{z_{r}}}=2 \frac{\partial^{2}}{\partial x_{0}^{2}}+\frac{1}{4} e_{1} \frac{\partial^{2}}{\partial x_{1}^{2}}+\frac{1}{4} e_{2} \frac{\partial^{2}}{\partial x_{2}^{2}}
$$

on an open set $\Omega$ in $\mathbb{R}^{3}$. 
Theorem 8. Let $\Omega$ be an open set in $\mathbb{R}^{3}$. If $f$ is a regular function on $\Omega$, then the following equation holds true:

$$
\square_{a} f(a)=-\frac{1}{8} \frac{\partial^{2} f(a)}{\partial A^{2}} .
$$

Proof. Since $f$ is a regular function on $\Omega$, we have the following system:

$$
\begin{gathered}
4 \frac{\partial u_{0}}{\partial x_{0}}=\frac{\partial u_{1}}{\partial x_{1}}+\frac{\partial u_{2}}{\partial x_{2}}, \frac{\partial u_{2}}{\partial x_{1}}=\frac{\partial u_{1}}{\partial x_{2}} \\
4 \frac{\partial u_{r}}{\partial x_{0}}=-\frac{\partial u_{0}}{\partial x_{r}} \quad(r=1,2) .
\end{gathered}
$$

By the definition of $\square_{a}$, we have

$$
\begin{aligned}
\square_{a} f= & \left(2 \frac{\partial^{2}}{\partial x_{0}^{2}}+\frac{1}{4} e_{1} \frac{\partial^{2}}{\partial x_{1}^{2}}+\frac{1}{4} e_{2} \frac{\partial^{2}}{\partial x_{2}^{2}}\right)\left(u_{0}+e_{1} u_{1}+e_{2} u_{2}\right) \\
= & 2 \frac{\partial^{2} u_{0}}{\partial x_{0}^{2}}+2 e_{1} \frac{\partial^{2} u_{1}}{\partial x_{0}^{2}}+2 e_{2} \frac{\partial^{2} u_{2}}{\partial x_{0}^{2}}-\frac{\partial^{2} u_{1}}{\partial x_{0} \partial x_{1}} \\
& +e_{1} \frac{\partial^{2} u_{0}}{\partial x_{0} \partial x_{1}}-\frac{\partial^{2} u_{2}}{\partial x_{0} \partial x_{2}}+e_{2} \frac{\partial^{2} u_{0}}{\partial x_{0} \partial x_{2}} \\
= & -2 \frac{\partial^{2} u_{0}}{\partial x_{0}^{2}}-2 e_{1} \frac{\partial^{2} u_{1}}{\partial x_{0}^{2}}-2 e_{2} \frac{\partial^{2} u_{2}}{\partial x_{0}^{2}}=-2 \frac{\partial^{2} f}{\partial x_{0}^{2}} .
\end{aligned}
$$

From Proposition 7, we have $\partial^{2} f / \partial A^{2}=4^{2}\left(\partial^{2} f / \partial x_{0}^{2}\right)$. Hence, by calculating and comparing the above polynomials, we obtain that $\square_{a} f$ is equal to $-(1 / 8)\left(\partial^{2} / \partial A^{2}\right) f$.

Next, we consider a differential form

$$
\omega=4 d x_{1} \wedge d x_{2}-e_{1} d x_{0} \wedge d x_{2}+e_{2} d x_{0} \wedge d x_{1}
$$

Theorem 9. Let $\Omega$ be an open set in $\mathbb{R}^{3}$ and let $U$ be any domain on $\Omega$ with a smooth distinguished boundary bU such that $U \subset \Omega$. If $f$ is a regular function on $\Omega$, then one has

$$
\int_{b U} \omega f=0
$$

where $\omega f$ is the reduced quaternionic product of the form $\omega$ on the function $f(a)$.

Proof. Since $\omega f=4 f d x_{1} \wedge d x_{2}-e_{1} f d x_{0} \wedge d x_{2}+e_{2} f d x_{0} \wedge d x_{1}$, we have

$$
\begin{aligned}
d(\omega f)= & 4 \frac{\partial f}{\partial x_{0}} d x_{0} \wedge d x_{1} \wedge d x_{2}+e_{1} \frac{\partial f}{\partial x_{1}} d x_{0} \wedge d x_{1} \wedge d x_{2} \\
& +e_{2} \frac{\partial f}{\partial x_{2}} d x_{0} \wedge d x_{1} \wedge d x_{2}
\end{aligned}
$$

$$
\begin{aligned}
= & 4 \frac{\partial\left(e_{1} f_{1}+e_{2} f_{2}\right)}{\partial x_{0}} d I+e_{1} \frac{\partial\left(e_{1} f_{1}+e_{2} f_{2}\right)}{\partial x_{1}} d I \\
+ & e_{2} \frac{\partial\left(e_{1} f_{1}+e_{2} f_{2}\right)}{\partial x_{2}} d I \\
= & \left\{\left(4 \frac{\partial u_{0}}{\partial x_{0}}-\frac{\partial u_{1}}{\partial x_{1}}-\frac{\partial u_{2}}{\partial x_{2}}\right)+e_{1}\left(4 \frac{\partial u_{1}}{\partial x_{0}}+\frac{\partial u_{0}}{\partial x_{1}}\right)\right. \\
& \left.+e_{2}\left(4 \frac{\partial u_{2}}{\partial x_{0}}+\frac{\partial u_{0}}{\partial x_{2}}\right)+e_{1} e_{2}\left(\frac{\partial u_{2}}{\partial x_{1}}-\frac{\partial u_{1}}{\partial x_{2}}\right)\right\} d I,
\end{aligned}
$$

where $d I=d x_{0} \wedge d x_{1} \wedge d x_{2}$ in $U$. From the corresponding Cauchy-Riemann system (39) for $f(a)$ in $\mathbb{C}(\mathbb{T})$, we have the system (56). Hence, $d(\omega f)=0$ and, therefore, by Stokes theorem, we obtain the following result:

$$
\int_{b U} \omega f=\int_{U} d(\omega f)=0 .
$$

\section{Conflict of Interests}

The authors declare that there is no conflict of interests regarding the publication of this paper.

\section{Acknowledgment}

The third author was supported by a 2-Year Research Grant of Pusan National University.

\section{References}

[1] A. S. Meglihzon, "Po povodu monogennosti kvaternionov," DoKlady Akademii Nauk SSSR 3, vol. 59, pp. 431-434, 1948.

[2] A. Sudbery, "Quaternionic analysis," Mathematical Proceedings of the Cambridge Philosophical Society, vol. 85, no. 2, pp. 199224, 1979.

[3] R. Fueter, "Die Funktionentheorie der Differentialgleichungen $\Delta u=0$ und $\Delta \Delta u=0$ mit vier reellen Variablen," Commentarii Mathematici Helvetici, vol. 7, no. 1, pp. 307-330, 1934.

[4] V. Soucek, Regularni funkce quaternionove promenne [Thesis], Charles University Prague, 1980.

[5] F. Sommen, "Monogenic differential forms and homology theory," Proceedings of the Royal Irish Academy A, vol. 84, no. 2, pp. 87-109, 1984.

[6] M. Naser, "Hyperholomorphic functions," Siberian Mathematical Journal, vol. 12, pp. 959-968, 1971.

[7] K. Nôno, "Hyperholomorphic functions of a quaternion variable," Bulletin of Fukuoka University of Education III, vol. 32, p. 2137, 1983.

[8] K. Nôno, "Characterization of domains of holomorphy by the existence of hyper-conjugate harmonic functions," Revue Roumaine de Mathématiques Pures et Appliquées, vol. 31, no. 2, pp. 159-161, 1986.

[9] K. Nōno, "Domains of hyperholomorphy in $C^{2} \times \mathbb{C}^{2}$," Bulletin of Fukuoka University of Education III, vol. 36, pp. 1-9, 1987.

[10] J. Ryan, "Complexified Clifford analysis," Complex Variables and Elliptic Equations, vol. 1, no. 1, pp. 119-149, 1982/83. 
[11] J. Ryan, "Special functions and relations within complex Clifford analysis. I," Complex Variables and Elliptic Equations, vol. 2, no. 2, pp. 177-198, 1983.

[12] H. Malonek, "A new hypercomplex structure of the Euclidean space $\mathbb{R}^{m+1}$ and the concept of hypercomplex differentiability," Complex Variables: Theory and Applications, vol. 14, no. 1-4, pp. 25-33, 1990.

[13] S. Gotô and K. Nôno, "Regular functions with values in a commutative subalgebra $\mathbb{C}(\mathbb{C})$ of matrix algebra $M(4, \mathbb{R})$," Bulletin of Fukuoka University of Education III, vol. 61, pp. 915, 2012.

[14] H. Koriyama, H. Mae, and K. Nôno, "Hyperholomorphic functions and holomorphic functions in quaternionic analysis," Bulletin of Fukuoka University of Education III, vol. 60, pp. 1-9, 2011.

[15] J. Kajiwara, X. D. Li, and K. H. Shon, "Regeneration in complex, quaternion and Clifford analysis," in Finite or Infinite Dimensional Complex Analysis and its Applications, vol. 2 of Advances in Complex Analysis and Its Applications, pp. 287-298, Kluwer Academic, Hanoi, Vietnam, 2004.

[16] J. Kajiwara, X. D. Li, and K. H. Shon, "Function spaces in complex and Clifford analysis," in Inhomogeneous Cauchy Riemann System of Quaternion and Clifford Analysis in Ellipsoid, International Colloquium on Finite or Infinite Dimensional Complex Analysis and Its Applications, vol. 14, pp. 127-155, Hue University, Hue, Vietnam, 2006.

[17] S. J. Lim and K. H. Shon, "Hyperholomorphic fucntions and hyperconjugate harmonic functions of octonion variables," Journal of Inequalities and Applications, vol. 77, pp. 1-8, 2013.

[18] S. J. Lim and K. H. Shon, "Dual quaternion functions and its applications," Journal of Applied Mathematics, vol. 2013, Article ID 583813, 6 pages, 2013.

[19] D. H. Titterton and J. L. Weston, "Strapdown inertial navigation technology," Peter Pregrinus, 1997.

[20] R. Fueter, "Die theorie der regularen funktionen einer quaternionenvariablen," in Comptés Rendus du Congrès International des Mathenaticiens, vol. 1, pp. 75-91, Oslo, Norway, 1936. 


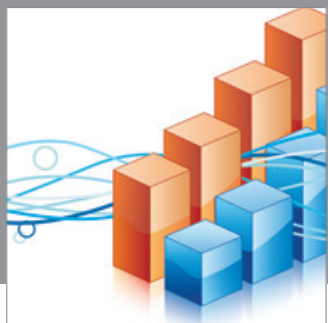

Advances in

Operations Research

mansans

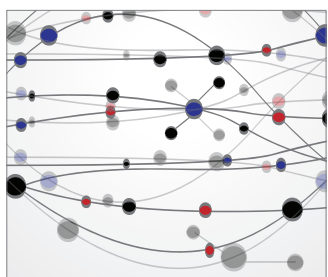

The Scientific World Journal
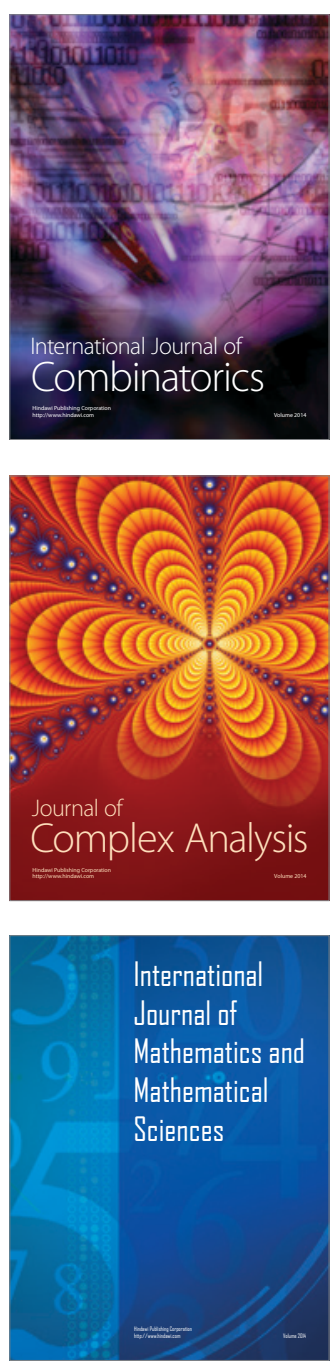
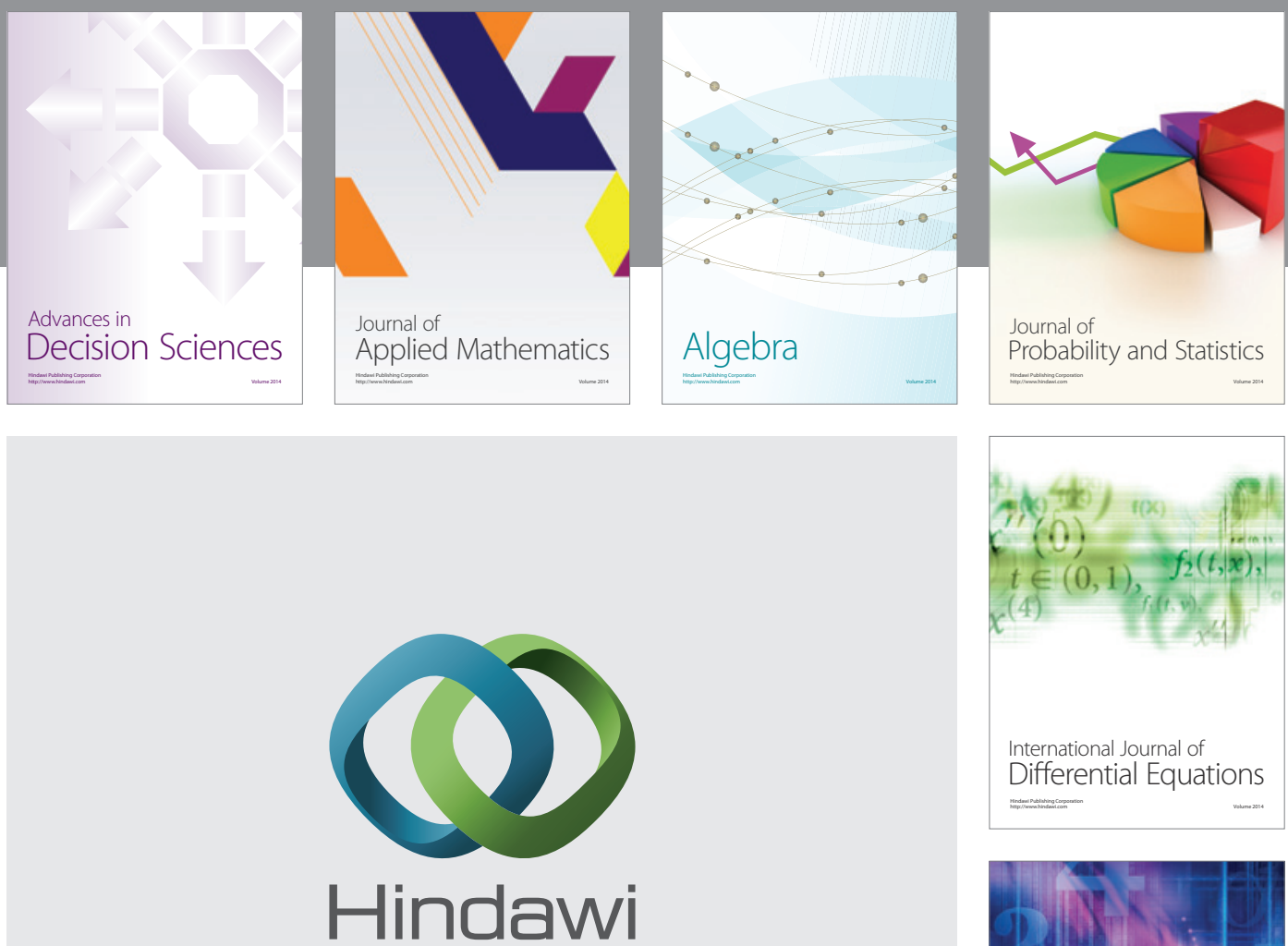

Submit your manuscripts at http://www.hindawi.com
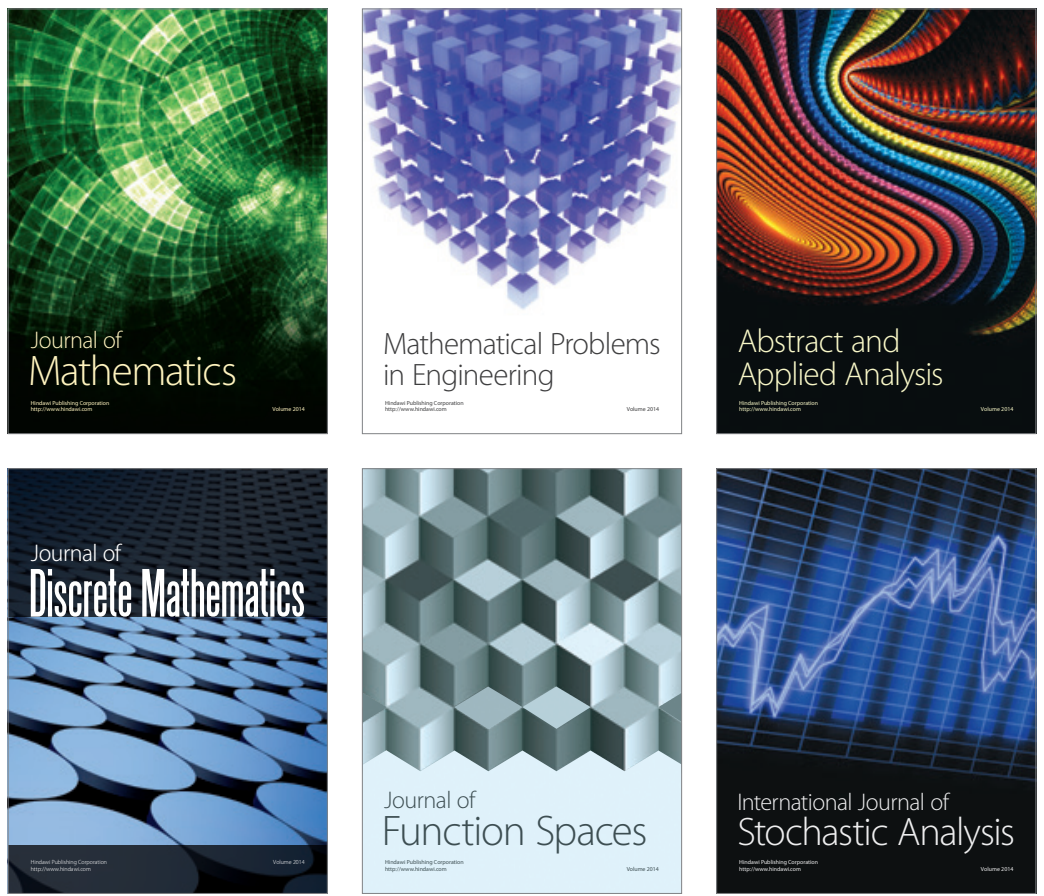

Journal of

Function Spaces

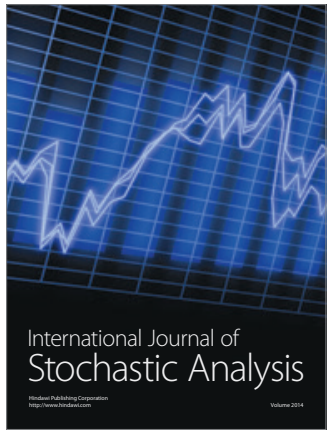

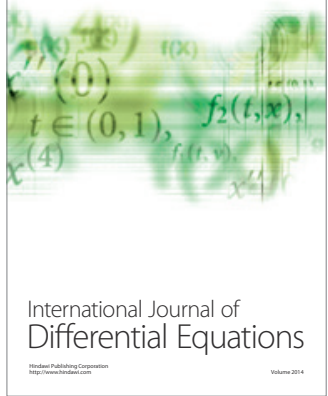
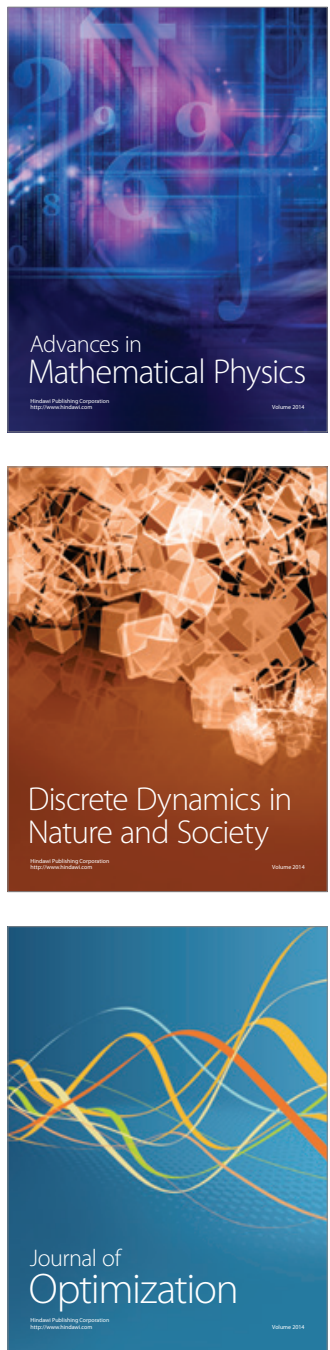